\title{
Temperature Changes of Kamaboko Accompanied by Stretching
}

\author{
Moritsugu Hamada, ${ }^{*}$ Takeshi Nagai, ${ }^{*}$ Norihisa Kai, \\ and Yasuhiro Tanoue* \\ *Department of Food Science and Technology, National Fisheries University, \\ Nagata-honmachi, Shimonoseki 759-65, Japan
}

(Received June 26, 1995)

The structure of elastic appearance of kamaboko was discussed by the thermodynamic theory of elastic deformation. The thermistor was inserted into kamaboko sample gels, and the temperature was measured as the gels were stretched at the rate of $20 \mathrm{~cm} / \mathrm{min}$. As a result, the temperature of the sample gels decreased right after the temperature increased. However, it is unlikely that a rapid variation of temperature of kamaboko occures in the atmosphere, because kamaboko will have almost the same magnitude of specific heat with that of water, and the cause for the temperature increase and decrease were examined. The increase in temperature when the sample gels were stretched was explained by the temperature distribution within the gels being low and high temperatures at the middle part and surface of the gels, respectively. On the other hand, the temperature decrease right after the temperature rise was likely caused by the heat of moisture vaporization when the surface area of the samples were expanded accompanied by stretching. The factors for temperature changes in kamaboko when it is stretched can be explained by factors other than the Gough-Joule effect. Therefore, it is not enough to conclude that kamaboko has an entropy elastic body only by the results from the apparent temperature increase.

Key words: Kamaboko, Elasticity, Gough-Joule effect, Entropy elasticity, Energy elasticity, Stretching

When food gels such as kamaboko, konjak mannan gel, agar and gelatin gels are stretched or twisted, their forms change greatly. However, when the force is loosened, their deformed shapes return to their original forms. Their elasticity is reported hitherto as entropy elasticity. ${ }^{1-6)}$ Meanwhile, we have conjectured that the elasticity of myosin gel is the energy elasticity by examining the temperature dependency of myosin gels. ${ }^{7-9)}$ It is very important to define whether the elasticity is entropy elasticity or energy elasticity, not only as an issue of interpretation, but also for clarifying the combination or existence of salt soluble proteins which compose kamaboko. This report will discuss the structure of elastic appearance in kamaboko by inspecting temperature changes when kamaboko is stretched in accordance with the thermodynamic theory of elastic deformation.

\section{Materials and Methods}

\section{Sample Gels}

One of the high grade kamaboko in a semi-circular shape on a piece of wood was purchased on the local market, and was used as a sample gel. These kamaboko products were prepared from frozen raw fish meat paste of walleye pollack Theragra chalcogramma, by heating in a gas oven.

\section{Measuring Apparatus}

A rheometer, type NRM-2000J, Fudoh Kogyo Co., was used to stretch the kamaboko. A thermistor with a globeshaped pointed head of $2.3 \mathrm{~mm}$ diameter, type
NTH2011C, Murata Seisakusho Co., was utilized to gauge temperature changes in sample gels when the gels were stretched. The differential thermistor thermometer had the same circuit as the device which Niwa et al. ${ }^{5}$ used. Also, a three terminal voltage regulator, type $78 \mathrm{~L} 06$, Nihon $\mathrm{Mu}$ sen Co. was combined anew to keep the voltage in the Wheatstone bridge circuit stable (Fig. 1). Moreover, as a comparison with stretched sample gel, non-stretched sample gel was also used, and the non-stretched sample gel was fixed very close to the stretched one. The thermistors 1 and 2 shown in Fig. 1 were inserted to the stretched sample gel and non-stretched one, respectively. The relationship between the height of the pen's vibration and the amount of the actual temperature change was counted on the relative equation which was examined independently by utilizing

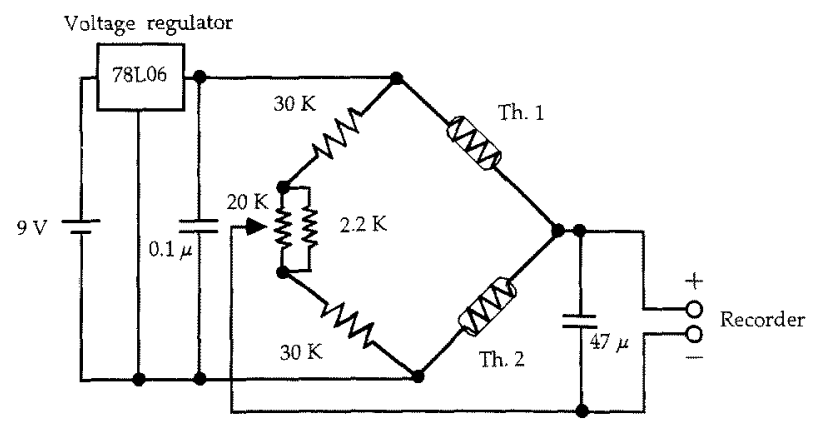

Fig. 1. Electric circuit for the differential thermistor thermometer. Thermistors 1 and 2 were inserted to the stretched gel and nonstretched one, respectively. 
Beckmann's thermometer.

\section{Measuring Conditions}

By using a specialized fixture, a sample of kamaboko gel with a size of $0.8 \mathrm{~cm}$ in width, $0.5 \mathrm{~cm}$ in height, and $3 \mathrm{~cm}$ in length, was mounted to the rheometer. It was then placed in a room where the temperature and relative humidity were regulated at $25^{\circ} \mathrm{C}$ and $45 \%$, respectively. A set of the sample gels and the rheometer were covered with a double hood which was made with acrylic resins with $0.215 \mathrm{~mm}$ in thickness. The hood was used to maintain the fixed temperature of the sample gels' periphery. The thermistors were inserted into the center of the sample gel and the reference gel, and both gels were let until the temperature became $\pm 0.001^{\circ} \mathrm{C}$. It usually took 1 to $2 \mathrm{~h}$ to complete this process. Then, the temperature changes of the gels were measured when the gel was being stretched with a speed of $20 \mathrm{~cm} / \mathrm{min}$, which was quite slower than that of Niwa et al. The tensility alternation, which is caused by stretching, was also recorded in a chart at the same time.

\section{Thermodynamics of Elastic Deformation}

When the unit elastic body is stretched, work per unit volume, $d W$, is given with the following equation, under the terms mentioned as follows; deformation amount is $d l$, the amount of stretching force is $f$

$$
d W=f d l
$$

Then, when the amount of change in the elastic body's internal energy is $d E$, the amount of entropy change is $d S$, and specific heat under a constant stain is $C_{l}=(d E / d T)_{l}$, the equation developed under adiabatic condition will be ${ }^{10)}$

$$
T / C_{l}(d f / d T)_{l}=-T / C_{l}(d S / d l)_{T}=(d T / d l)_{s}
$$

According to the equation (2), if $(d f / d T)$, is positive, $(d S /$ $d l)_{T}$ is negative and $(d T / d l)_{s}$ is positive. That is, in the materials whose stretching force increases with temperature under a fixed extension, the entropy of the materials decreases with stretching under a constant temperature, and the temperature rises with stretching at a adiabatic condition, or sudden extension. Rubber is a material which typically exhibits these reactions. Iron springs do not show these responses.

Therefore, if food gels are measured adiabatically, such as when examining for the possibility of heat generation when gels are stretched or measuring tensility changes when the stretched samples gels are heated, the structure of elastic revelation in each food can be clarified.

\section{Results and Discussion}

\section{The Temperature Changes when Kamaboko Sample Gels were Stretched}

The changes in tensility and temperature when sample gels were stretched independently with forces of 100 gramforce (gf), $200 \mathrm{gf}$ and $300 \mathrm{gf}$ are shown in Fig. 2. The largest tensility, $300 \mathrm{gf}$, was slightly lower than the force which causes breakage. As shown in this result, the temperatures of the gels rose as the stretching ratio increased. However, the temperature started dropping after rising, and the temperature descended to lower than the temperature of the original level. The alternation pattern of temperature shown here was partially the same as the pattern which Niwa et al. ${ }^{5)}$ gained, and it is regarded that the gels generate temperature accompanied by stretching. Niwa $e t$ $a l .{ }^{5)}$ concluded that the Gough-Joule effect was observed from the view of temperature rise in kamaboko accompanied by stretching. It also concluded that kamaboko is an entropy elastic body; however, regarding the interpretation of the temperature alternation pattern shown in Fig. 2 , some questions remain in the conclusion of Niwa et al. First of all, even if the temperature of the gels rise due to the Gough-Joule effect, it is still unclear why the temperature of gels which have high specific heat, suddenly descend. Secondly, it is also unclear why the temperature descended to a level which is lower than the temperature before stretching was started.

The following factors can be described as reasons for the temperature rise; 1) Gough-Joule effect; 2) frictional heat between the thermistor and gels when they are stretched; 3 ) because the temperature varies widely inside the gels, the thermistor passed through the high temperature region when stretching, or heat conduction from the high

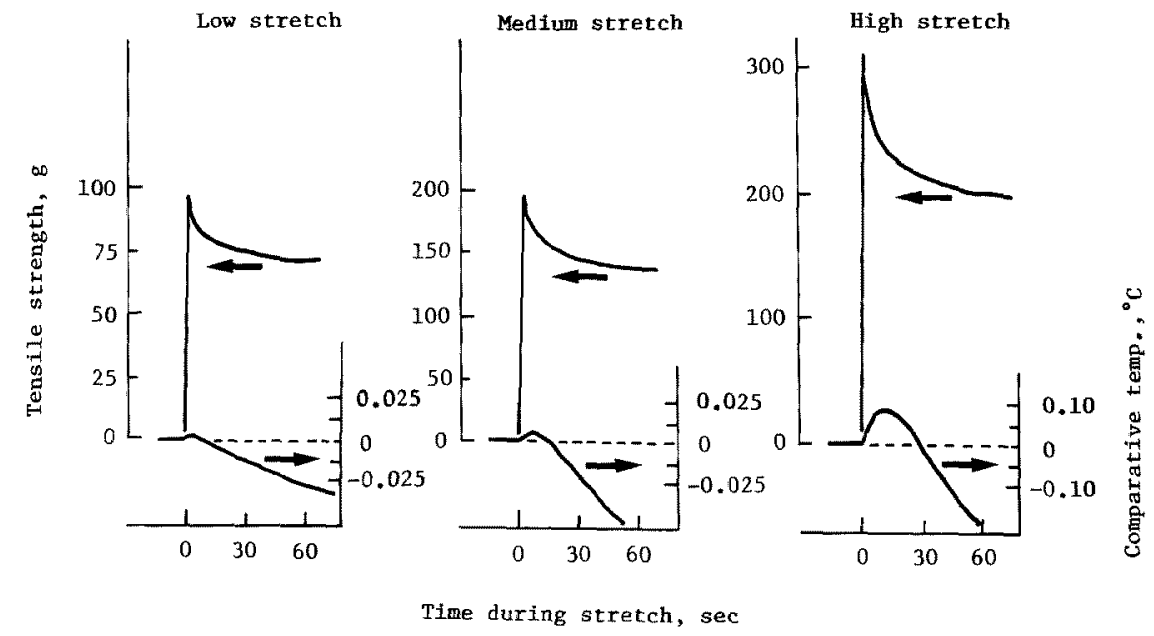

Fig. 2. Variations of tensile strength and temperature of kamaboko during stretch at the rate of $20 \mathrm{~cm} / \mathrm{min}$ at $25^{\circ} \mathrm{C}$. 
temperature region. On the other hand, the reasons why the temperature descends are; a) as the opposite theory of 3) above, after the thermistor passed through the higher temperature region, it reached the lower temperature region, or heat conduction from the low temperature region; b) the surface area of the sample gels expanded by stretching, and the temperature of the gels decreased due to loss of the heat of vaporization.

Then, the possibilities for 2) and 3) above regarding the temperature rise, and a) and b) for the temperature decrease were examined.

Possible Reasons for the Temperature Rise Accompanied by Stretching

To measure the temperature rise in kamaboko sample gels when stretched, stretching tests were performed after the signals from the thermometer had reached equilibrium. It took 1 to $2 \mathrm{~h}$ to complete the temperature equilibrium. Although the equilibrium was completed, there is a possibility that the temperature of the surface of the sample differed from the inside of the gels while the sample gels were left for 1 to $2 \mathrm{~h}$, because the thermistor was insert ed into only certain limited parts of the sample gels. After leaving the sample gels at $25^{\circ} \mathrm{C}$ for 1 to $2 \mathrm{~h}$, a stretching test was performed in order to measure relative rise in temperature of the limited part of the gel from the original temperature level. After completing the equilibrium of the sample's temperature with the usual method, the thermistor was gradually moved to the surface from the outside of the double hood by remote operation. After that, the thermistor was moved back to the original position, and the temperature changes were recorded as shown in Fig. 3. The surface temperature was higher than the temperature on the inside of the sample. Therefore, there are various temperature changes on the surface and inside of the gels, and the temperature distribution might have caused the

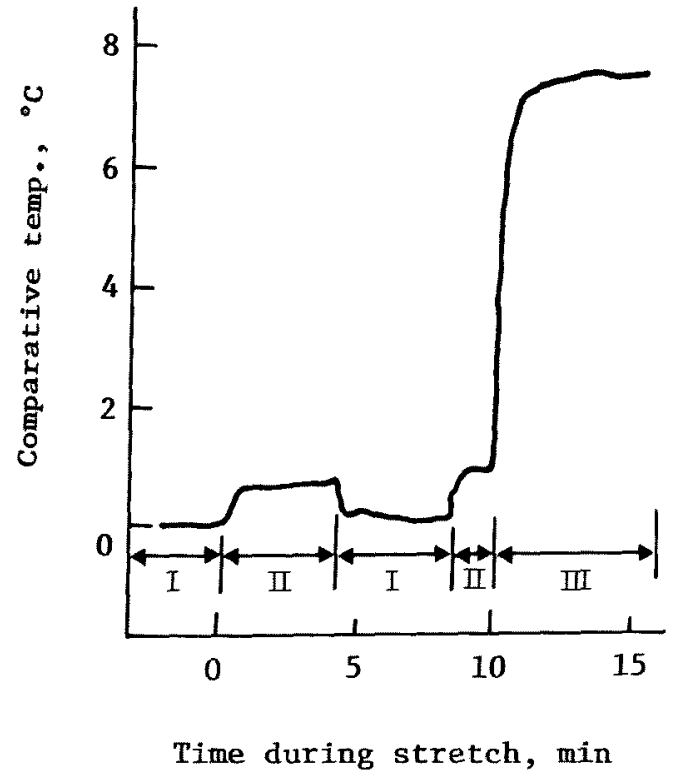

Fig. 3. Temperature of kamaboko and atmosphere.

I, inner part of the test piece of kamaboko; II, surface of the test piece; III, atmosphere. phenomenon of the temperature rise in the gels when stretching. That is to say, the following ideas made it easier to understand the temperature rise during stretching. The temperature of the gels was high on the surface and low inside. The part with high temperature made a contact with the thermistor when stretched as shown in Fig. 4.; as a result, the temperature of the sample gels looked as if it were rising. However, this theory cannot explain all the results which are shown in Fig. 2. Even if the thermistor detected temperature rise due to the effects from the higher temperature region, the theory mentioned above can not make clear the reasons for the temperature decrease right after the temperature increase, and for a temperature lower than the original temperature before stretching. Similarly, from the viewpoint of various temperatures in the gels, it was not enough to explain the phenomena of the temperature changes during stretching.

\section{Possible Reasons for the Temperature Decrease after Stretching}

There must be reasons for the sudden temperature decrease which was caused after the gels were stretched even though the temperature of the gels increased once. As a cause of the temperature decrease, there is a possibility that loss of the vaporization heat caused by the dehydrate from the gels' surface caused the phenomena. That possibility will be discussed here. When the gels are stretched by the greatest force, the surface areas of the gels become the widest. Then the moisture vaporation speed becomes the greatest, and the temperature decrease becomes the largest. Therefore, the possibility that these factors cause the temperature decrease during stretching gels was examined here.

To ensure the justification of this hypothesis, the following experimentation was performed. The original sample gels were lightly wrapped with polychlorovinylidene resin film, Hitachi Borden Co., and these gels were stretched after equilibrating temperature by leaving them in a constant temperature and humidity room for 1 to $2 \mathrm{~h}$ in accordance with the usual method. The reference sample gel was also wrapped as well at this time. The purpose of this experiment was to check the temperature changes of the gels accompanied by stretching, after equilibrating the gels' surrounding temperature with the surface temperature of the

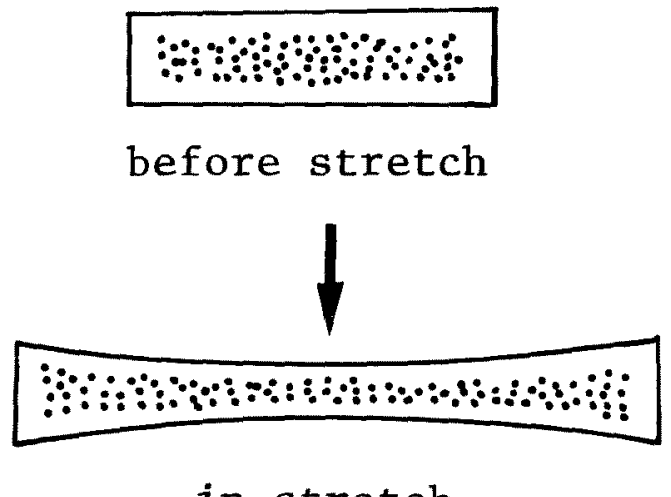

Fig. 4. A model for the temperature distribution of kamaboko. Dotted region indicates lower temperature region. 


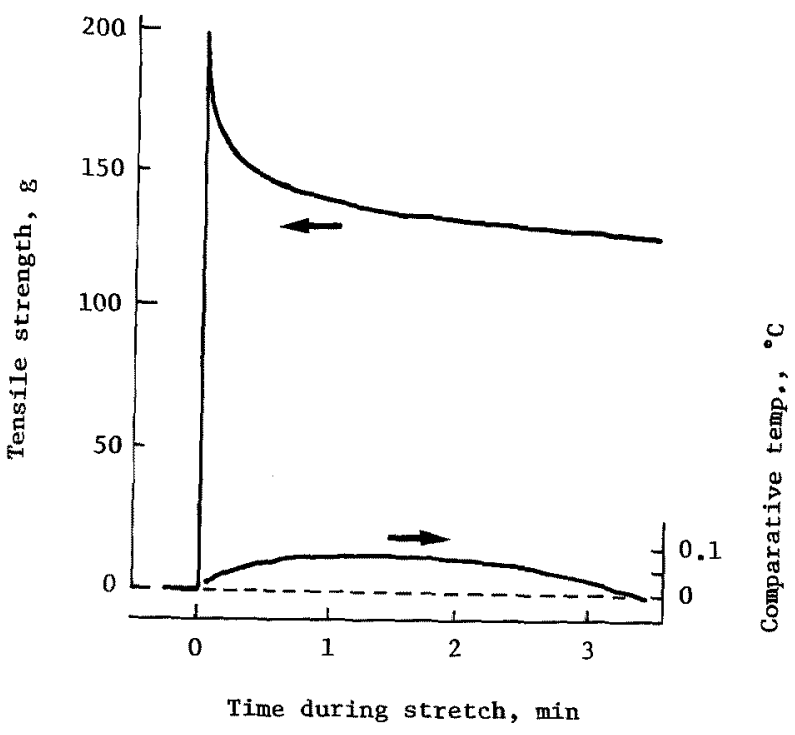

Fig. 5. Variations of tensile strength and temperature of kamaboko wrapped with polychlorovinylidene resin film.

gels. The temperature changes were as shown in Fig. 5.

As the results shown in Fig. 5, the temperature went up in accordance with the stretching, but the temperature began to decrease slightly after that. A drastic temperature decrease as shown in Fig. 2 was not shown here. Therefore, the temperature lowering in Fig. 2 is considered to be the heat of vaporization followed by moisture evaporation from the surface of the sample gel.

Although Niwa et al. defined the temperature increase at the time of stretching of gels as shown in Fig. 2 as the Gough-Joule effect, the possibilities that the temperature increased were caused by temperature distribution in the gel has been mentioned. Furthermore, it was mentioned that the cause for the temperature decrease right after the temperature increase may be attributed to the heat of vaporization accompanied with moisture evaporation from the surface of the samples.

\section{References}

1) Y. Ohta and K. Maekaji: Rheological properties of konjak mannan gel. Nippon Nögeikagaku Kaishi, 55, 415-419 (1981).

2) H. Nagura, H. Akabane, and N. Nakahama: Temperature dependence on the viscoelasticity of agar gel. Nippon Shokuhin Kogyo Gakkaishi, 31, 339-345 (1984).

3) I. Takagi and W. Shimidu: Thermodynamical components in elastic force of expanding kamaboko. Nippon Suisan Gakkaishi, 38, 475-479 (1972)

4) I. Takagi and W. Shimidu: Influence of starch contents upon thermodynamical components in elastic force of expanding kamaboko. Nippon Suisan Gakkaishi, 38, 769-772 (1972).

5) E. Niwa, E.-S. Chen, S. Kanoh and T. Nakayama: Entropy elasticity of food gels certified by Gough-Joule effect. Nippon Suisan Gakkaishi, 54, 249-252 (1988).

6) E. Niwa, E.-S. Chen, T.-T. Wang, S. Kanoh, and T. Nakayama: Extraordinarity in the temperature-dependence of physical parameters of kamaboko. Nippon Suisan Gakkaishi, 54, 1789-1793 (1988).

7) M. Hamada: Temperature dependencies of the rheological functions of fish myosin gel. J. Shimonoseki Univ. Fish, 39, 101-108 (1991).

8) M. Hamada, T. Nagai, N. Kai, and Y. Tanoue: Temperature dependence of the tensile strength of fish myosin gel. Fisheries Sci., 61, 464-466 (1995).

9) M. Hamada, T. Nagai, N. Kai, and Y. Tanoue: Variations of tensile strength of myosin gel by heating. Fisheries Sci., 61, 656-658 (1995).

10) T. Nakagawa: Rheology (in Japanese), in "Kindai-kogyokagaku" (ed. by Ryohei Oda et al.), Vol. 19, Asakura Shoten, Tokyo, 1969, pp. 236-243. 